\title{
BMJ Open Built environments for inpatient stroke rehabilitation services and care: a systematic literature review
}

\author{
Ruby Lipson-Smith (D) , ${ }^{1}$ Luis Pflaumer (D) , ${ }^{1}$ Marie Elf (D) , ${ }^{2}$ \\ Sarah-May Blaschke (D) , ${ }^{1}$ Aaron Davis (D) , ${ }^{1}$ Marcus White, ${ }^{3}$ Heidi Zeeman, ${ }^{4}$ \\ Julie Bernhardt (iD ${ }^{1}$
}

To cite: Lipson-Smith $\mathrm{R}$, Pflaumer L, Elf M, et al. Built environments for inpatient stroke rehabilitation services and care: a systematic literature review. BMJ Open 2021;11:e050247. doi:10.1136/ bmjopen-2021-050247

- Prepublication history and additional supplemental material for this paper is available online. To view these files, please visit the journal online (http://dx.doi. org/10.1136/bmjopen-2021050247).

Received 16 February 2021 Accepted 22 June 2021

\section{Check for updates}

C Author(s) (or their employer(s)) 2021. Re-use permitted under CC BY-NC. No commercial re-use. See rights and permissions. Published by BMJ.

${ }^{1}$ Stroke, Florey Institute of Neuroscience and Mental Health, Heidelberg, Victoria, Australia

${ }^{2}$ School of Education, Health and Social Studies, Dalarna University, Falun, Sweden ${ }^{3}$ Centre for Design Innovation, The Swinburne University of Technology, Hawthorne, Melbourne, Australia ${ }^{4}$ The Hopkins Centre, Menzies Health Institute Queensland, Griffith University, Meadowbrook, Queensland, Australia

Correspondence to Dr Ruby Lipson-Smith; ruby.lipson-smith@florey.edu.au

\section{ABSTRACT}

Objectives To identify, appraise and synthesise existing design evidence for inpatient stroke rehabilitation facilities; to identify impacts of these built environments on the outcomes and experiences of people recovering from stroke, their family/caregivers and staff.

Design A convergent segregated review design was used to conduct a systematic review.

Data sources Ovid MEDLINE, Scopus, Web of Science and Cumulative Index to Nursing and Allied Health Literature were searched for articles published between January 2000 and November 2020.

Eligibility criteria for selecting studies Qualitative, quantitative and mixed-methods studies investigating the impact of the built environment of inpatient rehabilitation facilities on stroke survivors, their family/ caregivers and/or staff.

Data extraction and synthesis Two authors separately completed the title, abstract, full-text screening, data extraction and quality assessment. Extracted data were categorised according to the aspect of the built environment explored and the outcomes reported. These categories were used to structure a narrative synthesis of the results from all included studies.

Results Twenty-four articles were included, most qualitative and exploratory. Half of the included articles investigated a particular aspect of the built environment, including environmental enrichment and communal areas $(n=8)$, bedroom design $(n=3)$ and therapy spaces $(n=1)$, while the other half considered the environment in general. Findings related to one or more of the following outcome categories: (1) clinical outcomes, (2) patient activity, (3) patient well-being, (4) patient and/or staff safety and (5) clinical practice. Heterogeneous designs and variables of interest meant results could not be compared, but some repeated findings suggest that attractive and accessible communal areas are important for patient activity and well-being.

Conclusions Stroke rehabilitation is a unique healthcare context where patient activity, practice and motivation are paramount. We found many evidence gaps that with more targeted research could better inform the design of rehabilitation spaces to optimise care.

\section{Strengths and limitations of this study}

- The review method allowed for all the current evidence regarding inpatient stroke rehabilitation built environments to be gathered and assessed in a systematic and rigorous way.

- The narrative synthesis and diagrams provide a succinct summary of the trends and gaps in stroke rehabilitation environments research.

- Results of the included studies could not be easily combined or compared due to heterogeneity of study designs and variables of interest.

- Stroke rehabilitation services vary globally, but the majority of the studies in this review were conducted in Australia ( $50 \%$ of included articles) and Sweden ( $21 \%$ of included articles).

PROSPERO registration number CRD42020158006.

\section{INTRODUCTION}

The physical environment of healthcare facilities can influence clinical outcomes, patient and staff experiences and the economic performance of the facility. ${ }^{1}{ }^{2}$ Healthcare design research generates evidence to inform the design of healthcare facilities. Recent healthcare design research has focused on acute environments such as surgery and intensive care, ${ }^{3}$ with significant attention paid to residential aged care ${ }^{4}$ and mental health facilities. ${ }^{5}$ Between these disparate sectors lies an important and expensive sector of healthcare: hospital-based inpatient rehabilitation.

Inpatient rehabilitation is essential for people recovering from serious injury or illness, such as stroke. ${ }^{6}$ Stroke is a leading cause of death and disability worldwide. ${ }^{7}$ As acute stroke treatments continue to improve, more people are expected to survive a stroke, and many will experience ongoing disability that requires hospital-based, or inpatient, rehabilitation. While recovery may continue for years poststroke, initial rehabilitation 


\begin{tabular}{|c|c|}
\hline Criteria & Eligibility requirements \\
\hline Publication year & Articles published between 2000 and 2020 (to reflect the rise of evidence-based design research in the past 20 years) \\
\hline Study design & $\begin{array}{l}\text { Quantitative, qualitative or mixed-methods research designs; excluded opinion pieces, commentaries, single case studies } \\
\text { and systematic reviews with no meta-analysis or meta-synthesis }\end{array}$ \\
\hline $\begin{array}{l}\text { Intervention or } \\
\text { phenomenon of } \\
\text { interest }\end{array}$ & $\begin{array}{l}\text { Detailed information about the built environment, including ambient features, architectural and landscape features, interior } \\
\text { design features and/or maintenance features; excluded articles that mentioned aspects of the built environment without } \\
\text { providing sufficient detail; for example, research that reported only the location of certain activities (eg, time spent in the } \\
\text { dining room) was not included, but research that provided details of said location (eg, dimensions and adjacencies) was } \\
\text { included }\end{array}$ \\
\hline
\end{tabular}

usually begins in the acute phase of care, followed by subacute inpatient rehabilitation for some and a gradual shift to outpatient and community care. Early supported discharge to home, more common in Europe, is suitable for only $30 \%$ of patients. ${ }^{68}$ The average length of stay in postacute inpatient stroke rehabilitation varies globally but is generally lengthy (eg, 27.2 days in Australia). ${ }^{9}$ There is evidence that functional outcomes vary between rehabilitation facilities. ${ }^{10}$ While variation may be due to differences in procedures and staffing, differences in environment could also contribute as we know that rehabilitation facility design is heterogeneous. ${ }^{11}$

Rehabilitation is defined as 'a process of active change by which a person who has become disabled acquires the knowledge and skills needed for optimum physical, psychological and social function'. ${ }^{12}$ Repetitive practice and targeted therapy-such as upper limb training, walking, speech exercises and practising activities of daily living-are integral to the rehabilitation process. People who have experienced a stroke are encouraged to engage in general physical, cognitive and social activities outside of their structured therapy time in order to further promote their recovery. ${ }^{13}$ This contrasts sharply with the priorities of acute care-to diagnose; stabilise the patient; and, where possible, apply acute treatments such as thrombolysis or clot retrieval to prevent death and optimise outcomes. ${ }^{14}$ During rehabilitation, patients must participate in activities and practice, but many patients experience boredom, lack of stimulation, fatigue, low mood and feelings of disempowerment, which negatively impact their motivation. ${ }^{15}$ The distinct function and priorities of rehabilitation, the importance of patient engagement and the typically long length of stay prompted this review of the healthcare design evidence specific to stroke rehabilitation to better understand how the design of these healthcare facilities could be optimised for their function.

The aim of this systematic literature review was to identify, appraise and synthesise the existing literature related to the design of inpatient stroke rehabilitation facilities. Our research questions were: What aspects of the built environment have been investigated in inpatient stroke rehabilitation settings? What types of research methods have been used? What types of outcomes have been investigated? What are the impacts of the built environment on the outcomes and experiences of patients recovering from stroke, their family/caregivers, and staff?

\section{METHODS \\ Design}

We aimed to include all relevant research, so we elected to conduct a mixed-studies systematic literature review, which followed the Preferred Reporting Items for Systematic Reviews and Meta-Analyses statement (see online supplemental file 1). ${ }^{16}$ We used a convergent segregated review design so that results from qualitative, quantitative and mixed-methods studies could be synthesised in a narrative summary. ${ }^{17}$ The protocol was prospectively registered on PROSPERO (date: 17 November 2019; see online supplemental file 2).

\section{Patient and public involvement}

An Advisory Committee including two stroke survivors reviewed the research questions and drafted the manuscript of this review.

\section{Data sources}

A systematic search was conducted in the following databases in January 2020 and updated in November 2020: Ovid MEDLINE, Scopus, Web of Science and Cumulative Index to Nursing and Allied Health Literature. A Boolean search strategy was used (see online supplemental file 3). Authors LP and RL-S searched the reference lists of included articles, systematic literature reviews, relevant PhD theses and key journals (Health Environments Research \& Design) and organisations (the Centre for Healthcare Design) for additional eligible studies. 


\section{Article selection}

Publications that met the criteria outlined in table 1 were considered eligible for inclusion. Following duplicate removal, two reviewers (RL-S and LP) independently screened the titles and abstracts of the remaining articles using Covidence. ${ }^{18}$ These authors then independently screened the full text of potentially eligible articles. Consensus was reached with whole team discussion.

\section{Quality appraisal}

Level of evidence and methodological quality were independently appraised by two reviewers. ${ }^{19}$ For level of evidence, RL-S and LP used criteria adapted from Stichler (see online supplemental file 4) ${ }^{2021}$ and reached consensus through discussion. Methodological quality was assessed using the Mixed Methods Appraisal Tool (MMAT). ${ }^{22}$ To ensure consistent use of the MMAT, $25 \%$ of the included articles were assessed collaboratively by RL-S and LP, before the remainder of the articles were independently assessed. Articles authored by reviewers were appraised by non-authors. Consensus was reached through discussion.

\section{Data extraction and synthesis}

Data were extracted using a standardised form (see online supplemental file 5). RL-S categorised the studies according to (1) the aspect of the built environment explored (eg, bedrooms) or approaches to altering the environment (eg, 'environmental enrichment'-that is, setting up a communal activity area, encouraging communal dining and providing patients with personalised 'enrichment packages' that include books, games and activities of their choice) and (2) the outcomes reported in findings. The categories were reviewed by authors and were used to structure the narrative synthesis. For the environmental enrichment articles included in this review, only the results pertaining to the built environment components of the enrichment intervention are discussed, namely, the availability and set-up of the communal activity areas.

\section{RESULTS}

After duplicate removal, our searches revealed 859 articles, 24 of which were included in the final review (see figure 1). These 24 articles reported 18 studies from 14 research groups and nine countries. We included only articles that focused on inpatient stroke rehabilitation healthcare built environments. Some articles were excluded because they were not specific to stroke rehabilitation $(n=14)$ or did not provide any details about the built environment $(\mathrm{n}=21)$.

The study characteristics, article focus, outcomes of interest, level of evidence and methodological quality of the 24 included articles are outlined in table 2, and their results are summarised in online supplemental file 6 . Half of the articles $(n=12)$ did not focus on a particular aspect of the built environment, instead exploring the impact of the built environment as a whole (see table 2). The remaining 12 articles investigated a particular aspect of the built environment, including environmental enrichment $(n=8)$,

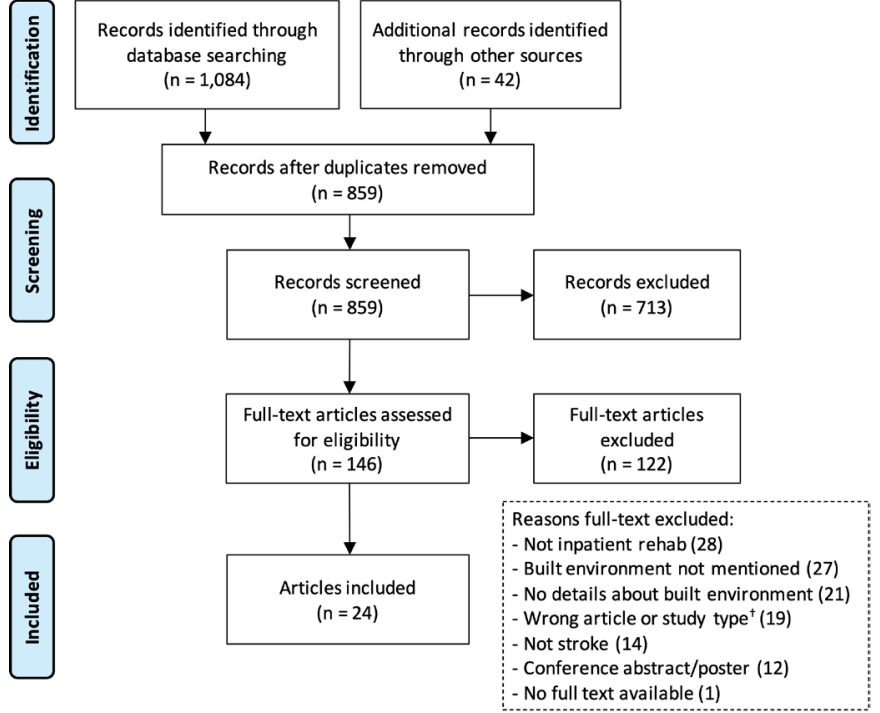

Figure 1 Article identification and screening flow diagram. tThe following types of articles were excluded from this review, but their reference lists were searched for relevant articles: opinion pieces or commentaries, unpublished studies in $\mathrm{PhD}$ theses, single case studies and systematic reviews with no meta-analysis, meta-synthesis or integrative component.

bedroom design $(n=3)$ and the location and availability of therapy spaces $(n=1)$. The aim of the environmental enrichment studies was to test, in humans, the long-established finding that laboratory rats who are housed with a rotating selection of toys, running wheels and other rats are more active and recover more effectively from brain injury than single rats in standard cages. ${ }^{23}$

In all included articles, one or more of the following five outcome categories were reported: (1) patient clinical outcomes (measurable changes in health or function, such as a person's balance, mobility or ability to perform everyday tasks), (2) patient activity (including physical, cognitive and/or social activities), (3) patient emotional well-being (including mood, boredom, loneliness, sense of empowerment and need for privacy), (4) patient and/or staff safety and (5) staff clinical practice and efficiency (such as clinical decision-making and use of staff time) (see table 2). These outcome categories are described in detail in the narrative synthesis below.

\section{Study design, research focus and methodological quality of the included articles}

Half of the included articles were qualitative studies $(n=12)$; the remainder were non-randomised quantitative studies $(n=6)$, mixed-methods studies $(n=5)$ and randomised quantitative studies $(n=1)$ (see figure 2 and table 2). In 18 of the 24 articles, patient outcomes or experiences were examined, rather than staff or family/ caregivers (see figure 2). In six articles, targeted research questions were addressed, e.g., prespecifying aspects of the built environment and/or specific outcomes of interest, while in other articles, a more exploratory approach was 


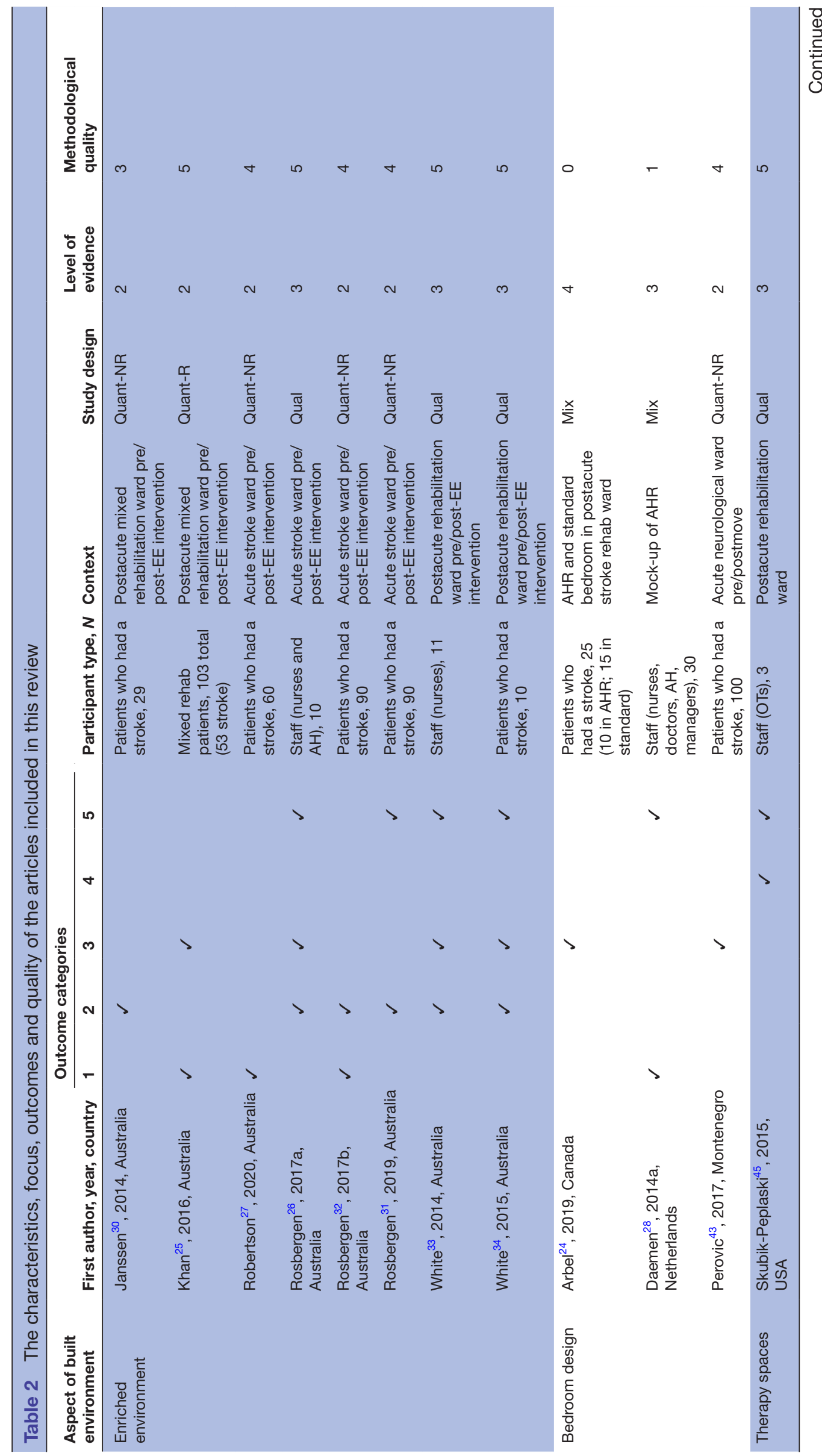




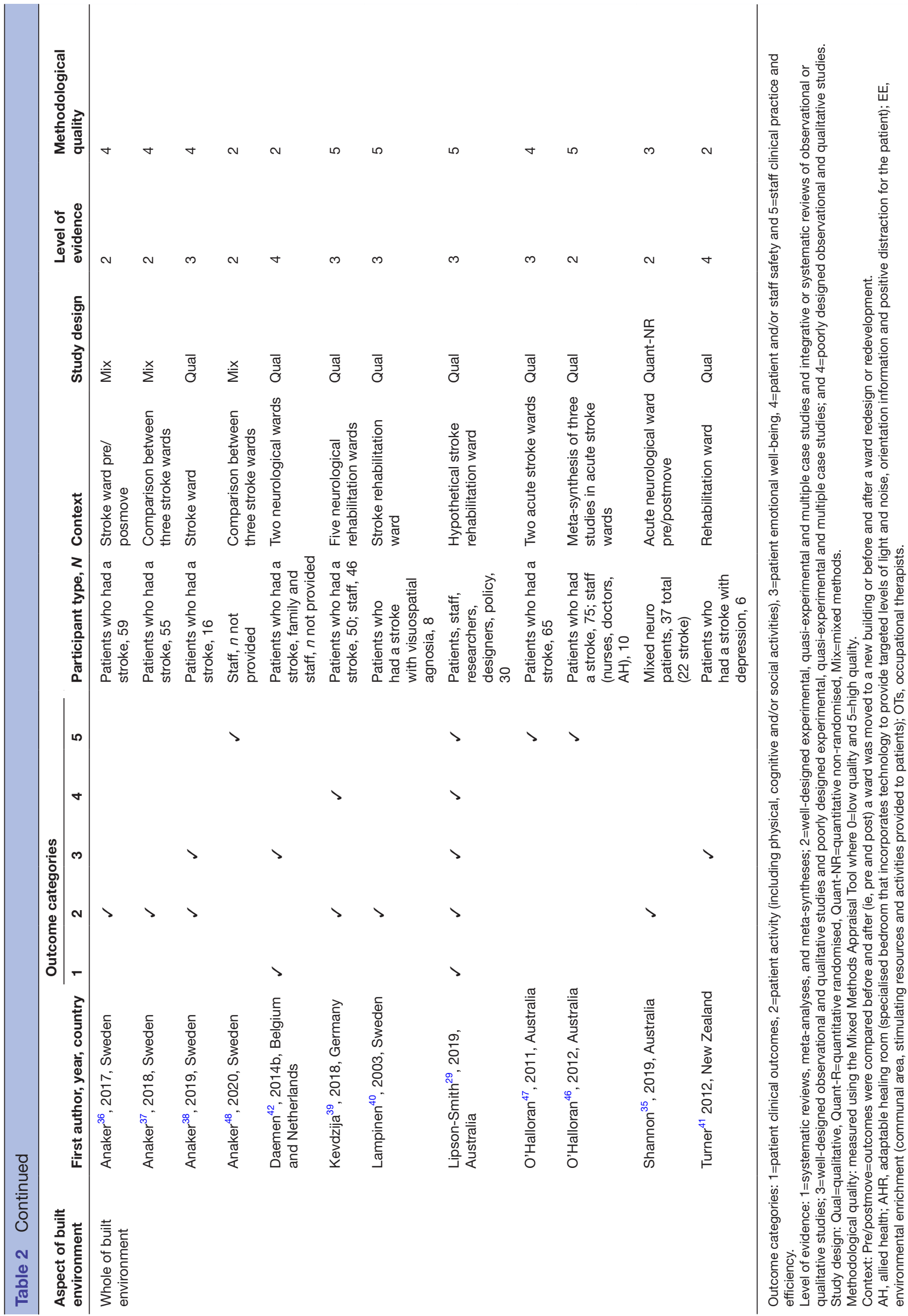




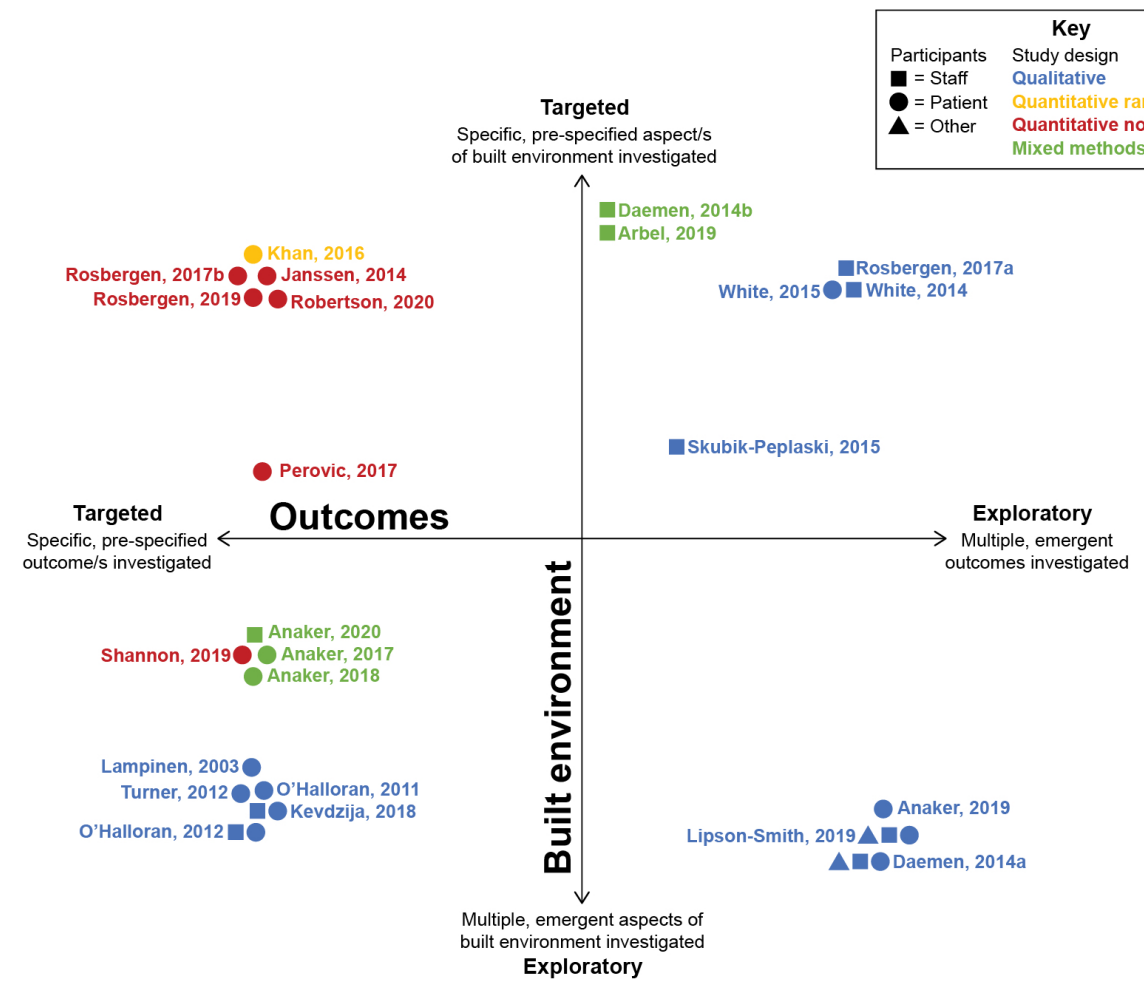

Figure 2 Research method and focus of included articles. Articles are clustered according to the extent to which they prespecified the specific aspects of the built environment or outcomes to be investigated (targeted vs exploratory research).

taken (see top left quadrant of figure 2). The role of the built environment in general was the focus in nine articles, in relation to specific outcomes of interest (lower left quadrant of figure 2), and the research questions in three articles were purely exploratory, with no predefined aspects of the built environment or outcomes of interest (bottom right quadrant of figure 2).

The qualitative studies appeared to be of higher methodological quality $(\mathrm{n}=12$, MMAT median score $=5)$, as did the one randomised quantitative study (MMAT score $=5$ ), while the non-randomised quantitative studies and mixed-methods studies were judged to be of lower methodological quality (non-randomised quantitative $\mathrm{n}=6$, MMAT median score $=4$; mixed methods $\mathrm{n}=5$, MMAT median score $=2$ ). Level of evidence classification is shown in table 2. All of the articles that received an MMAT score $<2$ (indicating low methodological quality) were also judged to provide the lowest level of evidence (level 4). The poorest scoring item on the MMAT was question 3.1 'Are the participants representative of the target population?' (see online supplemental file 7 ). We elected not to include one article ${ }^{24}$ in the narrative synthesis as it was assessed as having very low methodological quality (MMAT $=0$; see table 2 and online supplemental file 7 ).

\section{Narrative synthesis of results}

\section{Patient clinical outcomes}

In six articles (total $\mathrm{n}=263$ participants), one or more clinical outcomes were discussed (see table 2). Heterogeneity of outcomes, methods and environments prohibited comparison across studies.
In the only randomised trial, self-care and mobility functional independence at discharge from inpatient stroke rehabiliation were better in patients who had access to an enriched environment compared with patients without access (controls) ${ }^{25}$ Differences were not sustained at 3 months postdischarge; however, patients who experienced enrichment reported better health (measured using the EQ-5D) than controls. ${ }^{25}$ Fewer adverse events (such as worsening of symptoms) were reported in patients experiencing enrichment compared with controls in another study, with no difference in serious adverse events (such as hospitalisation or death) or malnutrition. ${ }^{26} 27$

One study explored staff opinion about the potential value of adaptable healing rooms (AHRs) for patients who had experienced a stroke. ${ }^{28}$ These specialised bedroom designs used timed lighting and multimedia technology to provide targeted levels of light and noise throughout the day, orientation information (eg, clock and timetable) and positive distraction (eg, family photos or nature scenes) for the patient. Staff suggested that AHRs may help to facilitate healing by promoting patient/staff relationships, being patient centred, helping patients to wake up naturally and improving sleep, providing more information and structure to the day and providing stimulation at the right times. ${ }^{28}$

Expert elicitation conducted with a large stakeholder group of people who had experienced a stroke and staff, researchers, architects, designers and policy makers ${ }^{29}$ revealed four agreed 'fundamentally important' 
objectives that the built environment should meet in order to optimise stroke rehabilitation care: maximising efficiency of care, maximising effectiveness of care (ie, clinical outcomes), maximising emotional well-being and maximising safety. The experts identified a number of 'instrumentally important' objectives that the built environment could achieve to maximise patient activity and effective sleep and rest and thereby maximise clinical outcomes, including maximising the versatility of the space, legibility (wayfinding), indoor environmental quality (air, light, noise, and so on) and patients' personal control over the space including accessibility to different spaces such as green and outdoor spaces and integration with the surrounding community. ${ }^{29}$

\section{Physical, cognitive and social activities}

In over half of the included articles (13 articles, total $\mathrm{n}=526$ participants), patient activity, including physical activity (eg, walking, using arm), cognitive activity (eg, reading, listening to music) and/or social activity (eg, talking, touch), was reported. Taken together, these studies provide some preliminary evidence that patient activity may increase in environments that: are legible and easy to navigate, have attractive and accessible communal areas and have a smaller proportion of single-bed patient rooms.

In two studies (reported across three articles), patients who had a stroke who were exposed to an enriched environment and a communal activity area participated in more activity than patients in a 'usual care' rehabilitation ward. ${ }^{263031}$ Variation in the type of activity enhanced with enrichment was found, with cognitive and social activities higher in one study, ${ }^{30}$ and physical, cognitive and social activities all found to be higher in the other study. ${ }^{2631} \mathrm{In}$ qualitative studies associated with these projects, both staff $^{3233}$ and patients ${ }^{34}$ reported that access to a communal activity area helped to promote patient activity.

In two studies, patient activity was measured before and after a ward was relocated to a new building. ${ }^{35}{ }^{36}$ In a further study, patient activity was measured across three existing wards. ${ }^{37}$ In these studies, a higher proportion of single-bed rooms was associated with lower levels of patient activity. Other aspects of the built environment thought to contribute to lower patient activity were the presence and attractiveness of communal areas and the ease of navigation. Communal areas that were unattractive or hard to find went unused. ${ }^{36-38}$

Kevdzija and Marquardt identified difficulty navigating (poor wayfinding), inappropriate dimensions of space (such as corridors that are too narrow for self-propelled wheelchairs), inappropriate distances between spaces (such as communal spaces being too far from the patient bedroom), uneven floor surfaces and physical obstacles (such as equipment left in corridors) as barriers. ${ }^{39}$ Similarly, legibility of the space, access to spaces beyond the bedroom (including communal and outdoor spaces) and patient control of the space were themes identified by Lipson-Smith et $a t^{29}$ during expert elicitation.
In a small qualitative study by Lampinen and Tham in which the challenges of agnosia (changes in ability to recognise objects) were specifically considered, participants described how unrecognisable objects in the environment became obstacles and created barriers to their activity and performance of everyday tasks. ${ }^{40}$

\section{Emotional well-being}

Emotional well-being was explored in nine articles in this review (total $\mathrm{n}=261$ participants). Patient mood, boredom, empowerment, privacy and loneliness were all raised as contributing to emotional well-being in inpatient rehabilitation. In several qualitative studies, communal area access appeared important for patient emotional wellbeing, reducing boredom and loneliness and promoting patient empowerment. ${ }^{29}$ 32-34 38 Reduced levels of depression, anxiety and stress at discharge were reported in patients with access to enrichment and communal areas compared with patients without access. ${ }^{25}$

Other built environment features thought to contribute to emotional well-being included flexible space (eg, having access to both single-bed and multibed patient rooms); connection to nature and the outside world; privacy and control over the space and allowing for personal spaces within a clinical environment; aesthetics and appropriate light and noise levels; and ease of navigation, legibility, and access within the space..$^{29} 384142$ In one quantitative study, no difference in depression or anxiety was found between patients in an old rehabilitation ward and those in a new rehabilitation ward, which had fewer beds per room, more natural light, more colour and a contemporary aesthetic. ${ }^{43}$

Staff and visitor/family emotional well-being were identified as important by Lipson-Smith et $a l^{29}$ but were not explored directly in any studies.

\section{Safety}

The concept of safety within the environment was addressed in only three studies (total $n=129$ participants). ${ }^{29} 3944$ In the study by Lipson-Smith et al, experts agreed that safety for patients, staff and visitors/family could be maximised by minimising manual handling; maximising sightlines between staff and patients; maximising legibility, accessibility and flexibility of the space; maximising indoor environmental quality (eg, light and noise); and incorporating modern technology. ${ }^{29}$ In a small qualitative study, occupational therapists felt safer treating patients in a gym environment than in one isolated and not purpose-built for therapy (such as a patient's bedroom) as there are always 'extra hands' available from fellow therapists in a gym. ${ }^{45}$ Obstacles in the environment (eg, equipment in the hallway) and uneven floor surfaces were perceived barriers to patient mobility in the study by Kevdzija and Marquardt. ${ }^{39}$ The actual safety, as opposed to perceived safety, of patients, staff and/or visitors was not measured in any of the included studies. 


\section{Clinical practice and efficiency}

Aspects of clinical practice and/or efficiency were mentioned in ten articles (total $\mathrm{n}=334$ participants). ${ }^{28} 29$ 31-34 45-48 In four articles, communal activity areas were explored in the context of staff workload. ${ }^{31-34}$ Staff opinion varied about whether communal areas increased staff workload; some nurses felt obliged to facilitate patients' use of the area, while other nurses felt that activity areas kept patients occupied and so decreased staff workload. ${ }^{32}{ }^{33}$ Quantitative studies in which staff time spent assisting patients in communal areas was measured suggested no change in staff workload when activity areas were introduced. ${ }^{31}$

An observational study of multiprofessional teamwork in three stroke units found that the design of the included stroke units did not appear to foster multiprofessional teamwork: Centrally located staff workplaces, such as the nurses' stations, created visible hubs but were not appropriate for confidential discussions between staff; none of the stroke units had dedicated rooms for multiprofessional meetings; and each profession worked mainly in their own dedicated offices. ${ }^{48}$

The qualitative meta-synthesis conducted by O'Halloran et $a l^{46}$ addressed the question of patient/staff communication and concluded that high levels of background noise, visual distractions and a lack of single-bed rooms acted as environmental barriers to communication between patients and staff. In another qualitative study, occupational therapists reported adapting their treatment sessions according to the available space, indicating that the suitability of therapy spaces impacts treatment decision-making. ${ }^{45}$

Finally, in studies by Lipson-Smith $e t a t^{29}$ and Daemon $e t$ $a l^{28}$, the role that the built environment, including AHRs, could play in contributing to care efficiency was raised in consultations with staff and other stakeholders.

\section{DISCUSSION}

This systematic review provides an overview of the existing research related to inpatient stroke rehabilitation built environments, a unique healthcare environment where patient activity, practice and motivation are paramount. Our review revealed a research field in its early stages; the majority of the included articles were exploratory (see figure 2), the quality of research varied and there was no research to provide level one evidence (see table 2). Heterogeneity of outcomes, methods and environmental variables of interest hindered comparison across studies but raised interesting questions about what drives research in this field and how this research is generated.

The few targeted research articles included in this review were limited to three aspects of the built environment: (1) environmental enrichment and associated communal activity areas; (2) bedroom design, including the impact of AHRs; and (3) the type and availability of therapy spaces. While these topics are important, they are hardly exhaustive. Access to nature and the outdoors was identified by Lipson-Smith et $a t^{29}$ as important for encouraging activity and emotional well-being in stroke rehabilitation environments, and the therapeutic impact of outdoor spaces is well researched in other healthcare settings, ${ }^{49}$ but our review revealed no targeted research studies addressing the impact of outdoor spaces in inpatient stroke rehabilitation.

Applying evidence-based design principles from other healthcare contexts to a rehabilitation setting is unlikely to fully address the unique priorities and purpose of rehabilitation environments. ${ }^{11}$ Single-bed patient rooms, for example, have been found to improve patient-clinician communication, infection control and noise reduction in other healthcare settings, ${ }^{50}$ but evidence regarding the impact of single-bed rooms is lacking in patients with neurological injury. ${ }^{51-53}$ Noise reduction and privacy are important considerations in stroke rehabilitation, especially considering the disabling experience of fatigue ${ }^{15}$; however, exploratory studies in this review suggest that patients in single-bed rooms may be less active and spend more time alone than patients in shared bedrooms, ${ }^{35} 36$ which may impact their recovery and well-being. ${ }^{29}$ More recently, Rosbergen et $a \tilde{l}^{4}$ found that patients spent more time alone but were also more physically active in a rehabilitation facility with more single-bed rooms, but there was no change in cognitive or social activity. Given the importance of both activity and rest in stroke rehabilitation, it is essential that the impact of single-bed rooms is further investigated in a rehabilitation-specific context so that a design solution can be achieved that facilitates activity and practice, while also providing opportunity for privacy and rest.

Communal areas were the most frequently addressed environmental feature in this review (addressed in half of the articles, $n=12$ ). Taken together, these articles allow some tentative conclusions to be drawn regarding the benefits of communal areas for patient activity and emotional well-being in stroke rehabilitation. This is in line with findings from a large qualitative study conducted in a general (not stroke-specific) rehabilitation setting, in which freedom of movement, access to facilities and choice within the environment impacted patient motivation, activity and social interaction..$^{55}$ Provision of communal dining and activity areas have also been noted as helping to increase patient activity. ${ }^{56}$ Importantly, the mere existence of a communal area is likely not sufficient to guarantee its use. ${ }^{37}$ Future research could examine the optimal design of communal areas including: whether their use should be flexible or structured, their optimal size and their optimal placement in relation to the patient bedrooms and other key spaces.

Patient perceptions and outcomes were the targets of interest in most studies (see figure 2). Variation in patient activity associated with the environment $(n=13)$ was explored in over half of the articles in this review. This is perhaps unsurprising since physical activity and fitness may predict outcomes after stroke. ${ }^{13}$ Healthcare environments can impact staff efficiency, well-being 
and retention, ${ }^{1}$ with flow-on effects for patient care. Family and caregiver involvement can improve patient outcomes,${ }^{57}$ yet caregivers often feel ignored or alienated in inpatient stroke rehabilitation environments. ${ }^{58}$ Future research should consider the impact of the built environment on staff and family/caregivers and how the environmental needs and priorities of these groups can be balanced with patients' needs.

Twenty-one articles were excluded from this review because, although they provided some comments about the built environment in their results or discussion, the authors did not intend to study the built environment and did not provide any details about said environment (see figure 1). For example, in some of these studies, the level of patient physical activity was shown to vary in different locations of the rehabilitation facility and be especially low in the bedroom and lounge. ${ }^{59}$ While these studies can help us understand, for example, high-use activity areas, the absence of details about the environment makes it impossible to determine in what way the environment is important.

This review showcases the wide array of study designs in this field. The authors of the one randomised study in this review acknowledged difficulties with conducting randomised trials of built environment interventions. This includes the inability to blind participants to randomisation outcome (because the environmental change is obvious), which can introduce bias. While Khan $e t a l^{25}$ found significant between-group differences with their enrichment intervention, they recommended the study be repeated in different settings with larger sample sizes to confirm their findings. In three studies, the authors took advantage of renovations or rebuilds to conduct comparative studies. While these natural experiments can be informative, rebuilds usually involve more than one design change and often coincide with significant procedural or social change in the healthcare service, making it difficult for environmental variables to be isolated. Standardised description of rehabilitation environments as well as replication of studies showing promising findings should be important goals for all healthcare built environments research. Innovative research approaches are needed to overcome the challenges of researching healthcare environments. Emergent research approaches in rehabilitation environments research include using virtual reality to model and test different designs in controlled experiment (eg, the Neuroscience Optimised Virtual Environment Living Lab Redesign Project, www. novellredesign.com).

The quality of the studies in this review varied according to the MMAT, with the qualitative studies achieving the highest scores (indicating higher quality). This may in part be a reflection of the scoring system used in the MMAT. The MMAT is, however, designed to be used for all study types, including mixed methods, and has precedent in healthcare environments research. ${ }^{1950}$ It is possible that our search may have missed some relevant research because the physical environment is defined differently in different disciplines, and some disciplines frequently publish in non-peer-reviewed mediums such as professional architecture magazines and books. However, we are confident that our search terms were sufficient to capture peer-reviewed research relating to the built environment as it is defined in this review. Our search was limited to articles published since the year 2000. We consider it unlikely that many relevant articles were published before this time. Indeed, only one $(4 \%)$ of the articles included in this review was published prior to 2010. The rate of research in this field is increasing; we are aware of relevant articles that are in preparation or that were published after our searches were completed. ${ }^{546061}$ This review should therefore be updated in the coming years.

The 24 articles in this review were produced by 14 research groups. Many of these groups have previously collaborated, and the authors of this review were involved in a number of the included studies. Evidence-based healthcare design research is inherently interdisciplinary, and the field will benefit as more diverse research groups bring innovative methods and approaches. The majority of the studies in this review were conducted either in Australia (50\% of included articles) or Sweden (21\% of included articles). As mentioned in the introduction, stroke rehabilitation services vary globally, and the design of rehabilitation facilities should reflect the local service. There is therefore a need to bring a more diverse international perspective to stroke rehabilitation environments research.

To effectively grow the research field and provide evidence-based design for patient well-being and health, it is essential that important factors (such as outdoor spaces, single-bed rooms, patient and staff safety and staff wellbeing) are not overlooked. We recommend that future researchers use the findings from the exploratory studies included in this review to provide a rationale and framework for their research in rehabilitation design. These exploratory studies identify aspects of the built environment and outcomes that are worthy of further investigation and provide a framework for future stroke rehabilitation environments research. This may encourage a more unified approach to the discipline and help researchers to identify aspects of the built environment and outcomes that are worthy of targeted study.

Twitter Ruby Lipson-Smith @RubyLipsonSmith and Julie Bernhardt @AVERTtrial

Acknowledgements This research was conducted on behalf of the Neuroscience Optimised Virtual Environment Living Lab (NOVELL) Redesign collaboration; see novellredesign.com for a full list of collaborators.

Contributors JB, ME, S-MB, MW and $\mathrm{HZ}$ conceived and planned the protocol for this study. S-MB developed and executed the search strategy. RL-S and LP assessed the articles for eligibility and conducted the data extraction. RL-S conducted the analysis and synthesis of the included studies. RL-S, LP, S-MB, ME, $\mathrm{AD}, \mathrm{MW}, \mathrm{HZ}$ and JB prepared the draft of the manuscript and edited, read and approved the final version of the manuscript.

Funding This research was funded by the Felton Bequest and the University of Melbourne as part of the Neuroscience Optimised Virtual Environment Living Lab (NOVELL) Redesign Project. JB is funded by an NHMRC Research Fellowship (1154904). The Florey Institute of Neuroscience and Mental Health acknowledges the support from the Victorian government and in particular the funding from the Operational Infrastructure Support Grant. 
Competing interests None declared.

Patient consent for publication Not required.

Provenance and peer review Not commissioned; externally peer reviewed.

Data availability statement All data relevant to the study are included in the article or uploaded as supplemental information. The results from the articles included in this systematic review are summarised in the supplemental material.

Supplemental material This content has been supplied by the author(s). It has not been vetted by BMJ Publishing Group Limited (BMJ) and may not have been peer-reviewed. Any opinions or recommendations discussed are solely those of the author(s) and are not endorsed by BMJ. BMJ disclaims all liability and responsibility arising from any reliance placed on the content. Where the content includes any translated material, BMJ does not warrant the accuracy and reliability of the translations (including but not limited to local regulations, clinical guidelines, terminology, drug names and drug dosages), and is not responsible for any error and/or omissions arising from translation and adaptation or otherwise.

Open access This is an open access article distributed in accordance with the Creative Commons Attribution Non Commercial (CC BY-NC 4.0) license, which permits others to distribute, remix, adapt, build upon this work non-commercially, and license their derivative works on different terms, provided the original work is properly cited, appropriate credit is given, any changes made indicated, and the use is non-commercial. See: http://creativecommons.org/licenses/by-nc/4.0/.

\section{ORCID iDs}

Ruby Lipson-Smith http://orcid.org/0000-0002-1702-8144

Luis Pflaumer http://orcid.org/0000-0001-9956-4639

Marie Elf http://orcid.org/0000-0001-7044-8896

Sarah-May Blaschke http://orcid.org/0000-0003-2737-3379

Aaron Davis http://orcid.org/0000-0002-1477-7406

Julie Bernhardt http://orcid.org/0000-0002-2787-8484

\section{REFERENCES}

1 Sadler BL, Berry LL, Guenther R, et al. Fable Hospital 2.0: the business case for building better health care facilities. Hastings Center Report 2011;41:13-23.

2 Ulrich RS, Zimring C, Zhu X, et al. A review of the research literature on evidence-based healthcare design. HERD 2008;1:61-125.

3 Joseph A, Bayramzadeh S, Zamani Z, et al. Safety, performance, and satisfaction outcomes in the operating room: a literature review. HERD 2018;11:137-50.

4 Chaudhury $\mathrm{H}$, Cooke $\mathrm{HA}$, Cowie $\mathrm{H}$, et al. The influence of the physical environment on residents with dementia in long-term care settings: a review of the empirical literature. Gerontologist 2017;58:e325-37.

5 Connellan K, Gaardboe M, Riggs D, et al. Stressed spaces: mental health and architecture. HERD 2013;6:127-68.

6 Norrving B, Barrick J, Davalos A, et al. Action plan for stroke in Europe 2018-2030. Eur Stroke J 2018;3:309-36.

7 World Health Organization. World health statistics 2019: monitoring health for the SDGs, sustainable development goals. Geneva: World Health Organization, 2019.

8 Langhorne P, Baylan S, Early Supported Discharge Trialists. Early supported discharge services for people with acute stroke. Cochrane Database Syst Rev 2017;7:CD000443.

9 Australasian Rehabilitation Outcomes Centre. The aroC annual report: the state of rehabilitation in Australia in 2019: Australian health services research Institute, University of Wollongong, 2019. Available: https://www.uow.edu.au/ahsri/aroc/dataset/reportsbenchmarks/

10 Reistetter TA, Kuo Y-F, Karmarkar AM, et al. Geographic and facility variation in inpatient stroke rehabilitation: multilevel analysis of functional status. Arch Phys Med Rehabil 2015;96:1248-54.

11 Lipson-Smith R, Zeeman H, Bernhardt J. What's in a building? A descriptive survey of adult inpatient rehabilitation facility buildings in Victoria, Australia. Arch Rehabil Res Clin Trans/ 2020;2:100040.

12 Bernhardt J, Hayward KS, Kwakkel G, et al. Agreed definitions and a shared vision for new standards in stroke recovery research: the stroke recovery and rehabilitation roundtable Taskforce. Int J Stroke 2017;12:444-50

13 Winstein CJ, Stein J, Arena R, et al. Guidelines for adult stroke rehabilitation and recovery: a guideline for healthcare professionals from the American heart Association/American stroke association. Stroke 2016;47:e98-169.
14 Powers WJ, Rabinstein AA, Ackerson T, et al. Guidelines for the early management of patients with acute ischemic stroke: 2019 update to the 2018 guidelines for the early management of acute ischemic stroke: a guideline for healthcare professionals from the American heart Association/American stroke association. Stroke 2019;50:e344-418

15 Luker J, Lynch E, Bernhardsson S, et al. Stroke survivors experiences of physical rehabilitation: a systematic review of qualitative studies. Arch Phys Med Rehabil 2015;96:1698-708.

16 Moher D, Liberati A, Tetzlaff J, et al. Preferred reporting items for systematic reviews and meta-analyses: the PRISMA statement. PLoS Med 2009;6:e1000097.

17 Lizarondo L, Stern C, Carrier J. Chapter 8: mixed methods systematic reviews. In: Aromataris E, Munn Z, eds. JBI manual for evidence synthesis. Adelaide, Australia: JBI, 2020.

18 Veritas Health Innovation. Covidence systematic review software. Melbourne, Australia: Veritas Health Innovation, 2020.

19 Taylor E, Hignett S. Evaluating evidence: defining levels and quality using critical appraisal mixed methods tools. HERD 2014;7:144-51.

20 Stichler JF. Weighing the evidence. Los Angeles, CA: SAGE Publications Sage CA, 2010.

21 Marquardt G, Motzek T. How to rate the quality of a research paper: introducing a helpful algorithm for architects and designers. HERD 2013;6:119-27.

22 Hong QN, Pluye P, Fàbregues S, et al. Mixed Methods Appraisal Tool (MMAT), version 2018. Registration of Copyright (\#1148552), Canadian Intellectual Property Office, Industry Canada. 2018.

23 Nithianantharajah J, Hannan AJ. Enriched environments, experiencedependent plasticity and disorders of the nervous system. Nat Rev Neurosci 2006;7:697-709.

24 Arbel I, Ye B, Mihailidis A. Stroke patients' experiences in an adaptive healing room in a stroke rehabilitation unit. Health Environments Research \& Design Journal 2019;13:170-85.

25 Khan F, Amatya B, Elmalik A, et al. An enriched environmental programme during inpatient neuro-rehabilitation: a randomized controlled trial. J Rehabil Med 2016;48:417-25.

26 Rosbergen IC, Grimley RS, Hayward KS, et al. Embedding an enriched environment in an acute stroke unit increases activity in people with stroke: a controlled before-after pilot study. Clin Rehabil 2017;31:1516-28.

27 Robertson ST, Grimley RS, Anstey C, et al. Acute stroke patients not meeting their nutrition requirements: investigating nutrition within the enriched environment. Clin Nutr 2020;39:1470-7.

28 Daemen EML, Flinsenberg ICM, Van Loenen EJ, et al. Adaptable healing patient room for stroke patients. A staff evaluation. Methods Inf Med 2014;53:406-15.

29 Lipson-Smith R, Churilov L, Newton C, et al. A framework for designing inpatient stroke rehabilitation facilities: a new approach using interdisciplinary Value-Focused thinking. HERD 2019;12:142-58.

30 Janssen H, Ada L, Bernhardt J, et al. An enriched environment increases activity in stroke patients undergoing rehabilitation in a mixed rehabilitation unit: a pilot non-randomized controlled trial. Disabil Rehabil 2014;36:255-62.

31 Rosbergen IC, Grimley RS, Hayward KS, et al. The impact of environmental enrichment in an acute stroke unit on how and when patients undertake activities. Clin Rehabil 2019;33:784-95.

32 Rosbergen ICM, Brauer SG, Fitzhenry S, et al. Qualitative investigation of the perceptions and experiences of nursing and allied health professionals involved in the implementation of an enriched environment in an Australian acute stroke unit. BMJ Open 2017;7:e018226.

33 White $\mathrm{JH}$, Alborough $\mathrm{K}$, Janssen $\mathrm{H}$, et al. Exploring staff experience of an "enriched environment" within stroke rehabilitation: a qualitative sub-study. Disabil Rehabil 2014;36:1783-9.

34 White $\mathrm{JH}$, Bartley $\mathrm{E}$, Janssen $\mathrm{H}$, et al. Exploring stroke survivor experience of participation in an enriched environment: a qualitative study. Disabil Rehabil 2015;37:593-600.

35 Shannon MM, Elf M, Churilov L, et al. Can the physical environment itself influence neurological patient activity? Disabil Rehabil 2019;41:1177-89.

36 Anåker A, von Koch L, Sjöstrand C, et al. A comparative study of patients' activities and interactions in a stroke unit before and after reconstruction-The significance of the built environment. PLOS One 2017;12:e0177477.

37 Anåker A, von Koch L, Sjöstrand C, et al. The physical environment and patients' activities and care: a comparative case study at three newly built stroke units. J Adv Nurs 2018;74:1919-31.

38 Anaker A, von Koch L, Heylighen A, et al. "It's Lonely": Patients experiences of the physical environment at a newly built stroke unit. Health Environments Research \& Design Journal 2019;12:141-52. 
39 Kevdzija M, Marquardt G, eds. Physical barriers to mobility of stroke patients in rehabilitation clinics. Cambridge workshop on universal access and assistive technology. Berlin: Springer, 2018.

40 Lampinen J, Tham K. Interaction with the physical environment in everyday occupation after stroke: a phenomenological study of persons with visuospatial agnosia. Scand J Occup Ther 2003;10:147-56.

41 Turner J. Environmental factors of hospitalisation which contribute to post-stroke depression during rehabilitation for over 65 year olds. Journal of the Australasian Rehabilitation Nurses' Association 2012;15:11-15.

42 Daemen E, van Loenen E, Cuppen R. The impact of the environment on the experience of hospitalized stroke patients - An exploratory study. In: European Conference on Ambient Intelligence 2014 Nov 11: Springer, Cham:114-24.

43 Perovic Z, Perovic S. Influence of hospital room environment on the reduction of anxiety and depression in the early stage of stroke. Journal of Environmental Protection and Ecology 2017;18:710-9.

44 Skubik-Peplaski C, Rowles GD, Hunter EG. Toward a physical environmental continuum for occupational intervention in a rehabilitation Hospital. Occup Ther Health Care 2012;26:33-47.

45 Skubik-Peplaski C, Howell DM, Hunter EG, et al. Occupational therapists' perceptions of environmental influences on practice at an inpatient stroke rehabilitation program: a pilot study. Physical and Occupational Therapy in Geriatrics 2015;33:250-62.

46 O'Halloran R, Grohn B, Worrall L. Environmental factors that influence communication for patients with a communication disability in acute hospital stroke units: a qualitative metasynthesis. Arch Phys Med Rehabil 2012;93:S77-85.

47 O'Halloran R, Worrall L, Hickson L. Environmental factors that influence communication between patients and their healthcare providers in acute hospital stroke units: an observational study. Int $J$ Lang Commun Disord 2011;46:30-47.

48 Anăker A, von Koch L, Eriksson G, et al. The physical environment and multi-professional teamwork in three newly built stroke units. Disabil Rehabil 2020:1-9.

49 Marcus CC, Sachs NA. Therapeutic landscapes: an evidence-based approach to designing healing gardens and restorative outdoor spaces. Hoboken: John Wiley \& Sons, 2013.
50 Taylor E, Card AJ, Piatkowski M. Single-Occupancy patient rooms: a systematic review of the literature since 2006. HERD 2018;11:85-100.

51 Shannon MM, Lipson-Smith R, Elf $M$, et al. Bringing the single versus multi-patient room debate to vulnerable patient populations: a systematic review of the impact of room types on hospitalized older people and people with neurological disorders. Intelligent Buildings International 2018:1-19.

52 Bernhardt J, Cumming T. The elephant in the single room debate: keeping patients active. BMJ 2013;347:f6333.

53 Maben J, Griffiths P, Penfold C, et al. One size fits all? Mixed methods evaluation of the impact of $100 \%$ single-room accommodation on staff and patient experience, safety and costs. BMJ Qual Saf 2016;25:241-56.

54 Rosbergen ICM, Tonello I, Clark RA, et al. Does Hospital design impact on patient activity levels and time spent alone? Disabil Rehabil 2020:1-8.

55 Killington M, Fyfe D, Patching A, et al. Rehabilitation environments: service users' perspective. Health Expect 2019;22:396-404.

56 Scrivener K, Pocovi N, Jones T, et al. Observations of activity levels in a purpose-built, inpatient, rehabilitation facility. Health Environments Research \& Design Journal 2019;12:26-38.

57 van Dijk M, Vreven J, Deschodt M, et al. Can in-hospital or post discharge caregiver involvement increase functional performance of older patients? A systematic review. BMC Geriatr 2020;20:362

58 Luker J, Murray C, Lynch E, et al. Carers' experiences, needs, and preferences during inpatient stroke rehabilitation: a systematic review of qualitative studies. Arch Phys Med Rehabil 2017;98:1852-62.

59 Sjöholm A, Skarin M, Churilov L, et al. Sedentary behaviour and physical activity of people with stroke in rehabilitation hospitals. Stroke Res Treat 2014;2014:163.

60 Janssen $\mathrm{H}$, Ada L, Middleton S, et al. Altering the rehabilitation environment to improve stroke survivor activity: a phase II trial. Int J Stroke 2021;17474930211006999:17474930211006999.

61 Kevdzija M, Marquardt G. Stroke patients' nonscheduled activity during inpatient rehabilitation and its relationship with the architectural layout: a multicenter shadowing study. Top Stroke Rehabil 2021:1-7. 